\title{
CONSTITUCIONALISMO \\ Y PROTECCIÓN PENAL DEL MATRIMONIO. \\ LA SECULARIZACIÓN DEL MATRIMONIO EN LA EVOLUCIÓN HISTÓRICA DEL ORDENAMIENTO PENAL ESPAÑOL* \\ Calvo Espiga, A.; Peñín González, Ma A. \\ Civitas y Thomson Reuters, Cizur Menor, 2010, 349 pP.
}

José A. Parody Navarro*

Nos hallamos ante una obra de denso y apretado contenido, como lo acreditan la riqueza del aparato crítico que figura a pie de página, el análisis de fuentes legislativas y jurisprudenciales y la amplia y selecta bibliografía con que se enriquece y sobre la que ha sido elaborada esta monografía.

Se abre el libro (pp. 13-27) con una ajustada y, a la vez, erudita introducción que describe perfectamente la línea metodológica que se seguirá en el tratamiento de la ardua cuestión que se han propuesto los autores. Cabe destacar la precisa presentación histórica que se realiza de la respuesta que el derecho ha dado a la institución matrimonial, desde que en las primeras compilaciones de leyes se planteó la importancia que para la sociedad tenía el matrimonio. Los autores, en esta Introducción, contextualizan de forma clara y precisa la problemática que se va a desarrollar y tratar a lo largo de su exposición.

Una vez situado el problema y los interrogantes que el mismo suscita, el estudio se articula en cuatro amplios capítulos. En el primero (pp. 29-138) se aborda el entorno constitucional, con una perspectiva jurídico-evolutiva, de la legislación matrimonial desde que, en el primer Código Penal, aparecen de forma sistemática normas protectoras del matrimonio. La novedad que presenta este capítulo, en el que se ofrece un detallado estudio de la experiencia constitucional española desde la de 1812, radica en el principio hermenéutico que se utiliza a la hora de analizar de la vertiginosa sucesión constitucional acaecida en España. La cronología se utiliza como cañamazo de un preciso estudio sistemático de aquellas normas constitucionales que, en los diversos textos, afectan al sistema penal y a la realidad del matrimonio. De gran interés

\footnotetext{
* Colaboración recibida el 30 de agosto y aprobada el 30 de septiembre de 2013.

** Doctor en Derecho, Profesor Colaborador Doctor Facultad de Derecho Universidad de Málaga, Málaga (España). Correo electrónico: japarody@uma.es.
} 
resultan, a este respecto, las páginas 131-138 en las que, consecuencia del método relacional-comparativo utilizado en el estudio de las Constituciones, se ofrecen las líneas dogmático-institucionales de evolución de la legalidad penal, según los distintos textos constitucionales, así como los principios penales que fueron progresivamente constitucionalizados a través del proceso histórico de elaboración y vigencia de las diferentes Constituciones. Ya en este primer capítulo resulta de gran interés la constatación de la dinamicidad y progresividad de la formulación y aceptación por el ordenamiento de una conformación del sistema penal en el que cada vez se tiene más en cuenta la protección de la persona y sus derechos sobre los de instituciones o corporaciones. En la obra, estos principios se sistematizan en torno a cuatro grupos: 1) en razón de las fuentes constitutivas o formalizadoras del Derecho Penal; 2) en razón de la determinación o tipificación de los caracteres del delito; 3 ) en cuanto a la tasación de las consecuencias del delito, y 4) en razón de la determinación de la jurisdicción y tipificación del proceso.

En el capítulo siguiente (pp. 139-239) se analiza el proceso mediante el que, por medio de la coerción de la norma penal, se ha dado cobertura y garantía a la institución matrimonial tal y como la delimitara en cada momento la legislación civil. Los autores abordan de forma directa el estudio de la protección penal que el ordenamiento español ha ofrecido a la institución matrimonial desde el Código Penal de 1822 hasta la codificación realizada en 1995.El método de análisis de Códigos y proyectos es de un gran rigor: bajo el pespunte de la historia, se realiza un preciso y exhaustivo estudio sistemático, dentro del contexto del ordenamiento penal en su conjunto, de la parte de la legislación penal que se refiere a la institución matrimonial. Entre otros aciertos, cabe destacar en este capítulo la Introducción al mismo (pp. 139-155) en la que se exponen los elementos y recursos que se van a utilizar para realizar un análisis sistemático y multidisciplinar de la evolución que la disciplina penal en torno al matrimonio ha experimentado, a lo largo de las distintas codificaciones o proyectos de codificación, en el ordenamiento español. Constituye en este estudio una absoluta novedad en el tratamiento que el Derecho Penal ha realizado de la institución matrimonial la presentación y análisis de las fuentes jurídicas del ordenamiento penal español. En concreto, se analiza El Codex repetitae praelectionis, El Fuero Juzgo, El Fuero Real, Las Partidas y La Novísima Compilación, escudriñando en ellas todos aquellos pasajes que hacen referencia a la protección jurídica de las variadas situaciones personales y sociales que afectan al matrimonio. Cabe notar que, a nuestro parecer, es la primera vez que, en la literatura doctrinal penal, se realiza un estudio del importante influjo que las fuentes jurídicas canónicas han tenido en el ordenamiento español. Se estudian éstas sistematizadas en tres grupos: 1) las anteriores al Decreto de Graciano, desde el siglo III hasta el XI; 2) el Decreto de Graciano, y 3) las Decretales de 
Gregorio IX. Más allá de la riqueza de la bibliografía utilizada por los autores, destaca el manejo y uso de forma directa y primaria de las fuentes jurídicas. No es fácil encontrar en la doctrina jurídica contemporánea referencias tan de primera mano a fuentes jurídicas fundamentales y absolutamente necesarias para el correcto conocimiento de nuestras instituciones jurídicas básicas. Llama la atención el cúmulo de horas que han debido dedicar los autores para la lectura y contraste de las fuentes jurídicas utilizadas, así como para elaborar la exquisita síntesis que de las mismas se nos ofrece; circunstancia perfectamente reflejada en la selecta riqueza del aparato crítico que avala todo juicio y afirmación que aparecen en el texto.

Entorno y principios constitucionales, tipo jurídico y cobertura penal sólo son efectivos en cuanto pueden ser aplicados y contrastados social y personalmente, bien en la dinámica implícita en el desarrollo normal del ordenamiento, bien en las situaciones de crisis y conflicto. La jurisprudencia es la dimensión del ordenamiento que contrasta socialmente y avala jurídicamente la medida y razón de esta efectividad. A ella dedican los autores el tercer capítulo (pp. 241305) de este estudio en el que desde el fondo constitucional, que determina las opciones legislativas concretas por un tipo u otro de matrimonio y avala hasta su protección penal, se ofrece no sólo el análisis del entorno normativo protector del matrimonio, sino el estudio de la íntima esencia jurídica de esta protección.

El capítulo tercero está todo él dedicado al análisis de la Jurisprudencia del Tribunal Supremo Español en torno a los delitos de posible comisión con relación al matrimonio. Si hasta ahora se ha puesto de relieve algunas de las aportaciones novedosas realizadas a la dogmática jurídica en los capítulos anteriores, hemos de notar que todo este capítulo constituye una absoluta novedad tanto por lo que se refiere a su contenido material como al tratamiento que del mismo se realiza. La Jurisprudencia del Tribunal Supremo analizada contiene sentencias que van desde el 14 de marzo de 1861 hasta el momento en que se elabora esta monografía.

En un primer apartado se analiza la Jurisprudencia generada a raíz de la oposición ejercida, tanto por particulares como por algunas autoridades, contra la Ley de Matrimonio Civil de 1870, siendo en este caso la primera sentencia estudiada de 5 de enero de 1874 al tiempo que, entre las estudiadas, se dedica una especial atención, por su relevancia jurídico-doctrinal, a la de 8 de febrero de 1896. A continuación se refiere la Jurisprudencia relacionada con la exigencia del consejo paterno, puesto que, como acertadamente señalan los propios autores, a pesar de una larga tradición canónica ya consolidada en la obra de Pedro Lombardo que privaba al consejo paterno de cualquier influencia en la validez del matrimonio, los ordenamientos civiles habían conservado, sin duda alguna influenciados por el derecho histórico francés, la exigencia de este 
requisito incluso bajo pena de nulidad. En un nuevo apartado se presenta la doctrina jurisprudencial emanada en torno a la situación creada por las viudas que contraían matrimonio sin observar los plazos legalmente previstos entre la defunción del marido y el nuevo matrimonio, siendo la primera sentencia estudiada de 6 de julio de 1876.

En estricta consecuencia con el propio material que ofrecen las sentencias del Tribunal Supremo, la parte más importante del análisis jurisprudencial se dedica en esta obra al tratamiento que el Alto Tribunal realiza del delito de bigamia. Siempre desde la estricta perspectiva del contenido ofrecido por las sentencias del Supremo, se estudia la delimitación del tipo penal tal y como se perfila en los pronunciamientos del referido Tribunal: la primera sentencia analizada es del 1 de abril de 1882. A continuación se presenta, tal como viene delimitado por la Jurisprudencia, el contenido del bien jurídico protegido mediante el delito de bigamia, también denominado matrimonio ilegal. En los apartados siguientes se realiza un sintético análisis de la problemática planteada, en relación al delito de bigamia, por los matrimonios civiles celebrados durante la Guerra Civil española en la zona republicana; el tratamiento que la Jurisprudencia realiza de la incidencia del dolo en la bigamia; la cualificación jurídica de la falsedad en la bigamia: si se trata de un nuevo delito o de un simple medio de prueba, como ya se planteara el Auto del TS, de 26 de noviembre de 1890; y, finalmente, el modo como la Jurisprudencia ha resuelto el tema de la prescripción del delito de bigamia. Desde la primera sentencia analizada (1861) se aprecia y demuestra una constante tendencia jurisprudencial a reducir a mínimos el recurso a la sanción penal como recurso de protección y salvaguarda de la institución matrimonial, buscando, también en lo que al delito de bigamia se refiere, más la seguridad jurídica de las personas casadas o en trance de contraer matrimonio que la incolumidad del matrimonio.

El cuarto y último capítulo (pp. 307-323), de carácter netamente conclusivo, pone de relieve, por una parte, la dinámica secularizadora que ha seguido la norma penal protectora del matrimonio en el ordenamiento español; $y$, por otra, la progresiva tendencia de la ley a proteger el ejercicio personal de un derecho, más que las propiedades o la propia existencia de la institución matrimonial en cuanto tal. Los autores nos ofrecen una recopilación perfectamente ajustada a la exposición que antecede, al tiempo que sintetizan con gran precisión las ideas fundamentales que sistemáticamente han sido desarrolladas a lo largo de todo el texto. De nuevo llama en ella la atención la densidad de su redacción y la profundidad y claridad con que se manejan conceptos jurídicos de compleja comprensión.

No resulta fácil encajar, dentro del entorno del género académico recensión, un comentario que abarque todos y cada uno de los elementos tan positivos que pueden ser destacados en esta obra. Lo primero que llama poderosamente 
la atención es la unidad de estilo redaccional que se observa en toda ella, al hallarnos, además, ante una redacción ágil, fluida y de cómoda lectura que, sin embargo, conserva y transmite una gran profundidad jurídica en el tratamiento de todos los problemas que se plantean. El esfuerzo de coordinación en la estructura literaria del libro para evitar rupturas en la lógica y desarrollo unitario de la exposición dice mucho de la calidad del trabajo con que se ha elaborado esta monografía. Simplemente ojeando el índice de esta obra se recibe la impresión de que el análisis que en ella se ofrece es una especie de elaborada seda jurídica, tanto por la finura de su método como por la precisión y acierto en la elección y utilización del material jurídico sobre el que se trabaja. Por otra parte, sería prolijo hacer referencia a todas y cada una de las aportaciones innovadoras y novedosas, desde el punto de vista de la protección penal del matrimonio, que aporta esta monografía. Nos limitaremos en este comentario a señalar algunas de ellas.

Sin duda alguna, el libro que presentamos constituye una importante aportación al Derecho Eclesiástico del Estado así como a la dogmática penal contemporánea, en la que, una vez más, hemos de constatar el dominio de la doctrina y jurisprudencia que manifiestan sus autores, tanto por lo que se refiere a la dimensión histórica de las mismas como a las más recientes aportaciones sobre el tema. Mérito a resaltar que, además, supone una auténtica novedad y relevante aportación al estudio del matrimonio es la transversalidad conceptual entre diversas materias jurídicas y la interdisciplinariedad presentes en toda la exposición. En el desarrollo de las distintas cuestiones tratadas se armonizan, dentro de la complejidad que supone el estudio de cualquier institución jurídica de la extensión y dificultad del matrimonio, contenidos y métodos de distintas disciplinas y especialidades jurídicas, desde el Derecho Constitucional al Penal, desde la Historia a la Jurisprudencia, desde el Derecho Canónico al Procesal, etc. Nos hallamos, por tanto, ante una de esas obras que, por la propia relevancia del asunto tratado, por la precisión, hondura y exhaustividad de sus análisis y por la riqueza y amplitud de las fuentes utilizadas, están Ilamadas a una permanencia, utilidad y validez que sobrepasan los límites de la actualidad de su publicación. Quien, a partir de esta monografía, intente el estudio de esta parcela del ordenamiento jurídico tiene roturado y recorrido un camino seguro y un punto de llegada absolutamente contrastado desde el que avanzar por otros derroteros en la certeza de que con esta obra se halla ante una firme y científicamente contrastada adquisición en el conocimiento de la institución matrimonial.

No resta sino hacer propias las palabras con las que el editor, en contraportada, presenta y valora esta monografía: "Tanto el método utilizado en este estudio como el tratamiento y manejo de fuentes jurídicas, tanto históricas como vigentes, que en él se realiza constituyen una auténtica novedad en el panorama 
bibliográfico español. Del mismo modo, supone una importante aportación a la reflexión jurídica la constatación del fenómeno secularizador y privatizador que se ha operado en el tratamiento de la institución matrimonial por parte del ordenamiento español: de una norma protectora de la institución se ha pasado a unas leyes protectoras de la persona y del ejercicio de sus derechos. Es de destacar, en esta monografía, como ya se ha apuntado, la modélica utilización de fuentes jurídicas tanto históricas como contemporáneas, así como la amplitud y cuidada selección de la bibliografía consultada y analizada. Constituye, por ello, una obra de referencia obligada tanto para los profesionales del foro como para los estudiosos y operadores jurídicos, ya se dediquen al estudio o práctica del derecho penal o bien al derecho matrimonial y de familia. La presente obra ofrece al lector tal riqueza jurisprudencial, de fuentes jurídicas y bibliografía que la convierte en instrumento indispensable para todo aquel que busque conocer en sus fundamentos e historia la institución matrimonial". 